\title{
Characterization of fouling layers for in-line coagulation membrane fouling by apparent zeta potential
}

Jie Wang*ab, Sasa Yang ${ }^{\mathrm{b}}$, Wenshan Guo ${ }^{\mathrm{c}}$, Huu-Hao Ngo ${ }^{\mathrm{c}}$, Hui Jia ${ }^{\mathrm{a}}$, Jun Yang ${ }^{\mathrm{b}}$, Hongwei Zhang $^{\mathrm{a}}$ and Xinbo Zhang ${ }^{\mathrm{d}}$

${ }^{a}$ State Key Laboratory of Separation Membranes and Membrane Processes, Tianjin Polytechnic University, Tianjin 300387, China. E-mail:wangjie@tjpu.edu.cn; Fax: +86 0228395 5668; Tel: +86 02283955668

${ }^{b}$ School of Environmental and Chemical Engineering, Tianjin Polytechnic University, Tianjin 300387, China

${ }^{c}$ Centre for Technology in Water and Wastewater, School of Civil and Environmental Engineering, University of Technology Sydney, Sydney, NSW 2007, Australia

${ }^{d}$ School of Environmental and Municipal Engineering, Tianjin Chengjian University, Tianjin 300387, China

This study investigated the apparent zeta potential of fouled membranes for in-line coagulation membrane fouling monitoring in micro-polluted water treatment. Results show that the apparent zeta potentials are consistent with transmembrane pressures (TMPs) in both a direct filtration process and in-line coagulation ultrafiltration (C-UF). Furthermore, the curve between apparent zeta potential and filtration resistance of C-UF conformed to the form of the Michaelis-Menten equation. The changes of apparent zeta potential along with periodical backwashing were in accordance with the trend of TMP change. As a whole, apparent zeta potential could be a useful indicator for monitoring membrane fouling.

\section{Introduction}

Microfiltration (MF) and ultrafiltration (UF) membrane processes have been widely used in micro-polluted water treatment to remove micro-organisms, particles and colloidal species. However, membrane fouling, especially fouling caused by the deposition on the membrane surface during filtration is a major obstacle for wide application of these processes. ${ }^{1-3}$ Hence, coagulation pretreatment for removing the colloidal matter is indispensable before MF or $\mathrm{UF}^{3-5}$ The dead-end filtration mode, which has been extensively adopted in micro-polluted water treatment, is the most economical from the viewpoint of energy. ${ }^{6}$ Nevertheless, this 
mode is particularly prone to generate a fouling layer. The complex fouling layer formed during filtration caused TMP increase, which depends on the properties of the fouling layer.

In order to analyze the structure characteristics of the fouling layer during filtration, lots of in situ techniques have then been proposed. Li et al. ${ }^{7,8}$ proposed a visualization ultrasonic time domain reflectometry for in situ investigation of fouling behavior in submerged hollow fiber membrane module under sub-critical flux. The measurements proved dynamic deposition behavior of gradual migration from top to bottom on the surface of membrane. Bérubé et al. ${ }^{9}$ quantified the shear at the surface of submerged hollow fiber membranes in different operation conditions by establishing the electrochemical shear probe. The results showed that the surface shear force increased along the length of the fiber in the direction of the bulk flow. Moreover, a previously developed optical method was used in situ to study time variations of the local thickness and porosity of depositions. ${ }^{6,10}$ The local characterization was performed during dead-end UF in a confined geometry resembling in/out hollow fibers with regard to the global operating parameters (flux and pressure). The studies provided a better understanding of the relationship between deposit structure and process performance. Hughes et al. ${ }^{11}$ introduced femtosecond near infrared non-linear optical imaging technique as a non-invasive in situ optical technique for 3D imaging of filtration cakes during cross-flow microfiltration. Based on the 3D femtosecond images, it was possible to measure the thickness of the filter cake formed on the MF membranes and identify fine structural features.

Zeta potential is an important parameter in fouling studies as it provides useful information about the charge properties of the membrane surface in solution. ${ }^{12,13}$ Thus, zeta potential was proposed to explain membrane retention mechanism and fouling properties of the fouling processes, which are strongly influenced by the surface charge of the membrane in contact with the feed water containing colloidal matters. ${ }^{14,15}$ In addition, membrane performance depends on interactions of membrane-fouling layer, membrane and layer morphology and their electrochemical properties. ${ }^{16}$ Electro-kinetic properties together with fouling resistance analysis can provide useful information for comprehension of fouling, because the fouling layer is a key point for understanding fouling and membrane performance. Therefore, there is a need of methods to characterize the electro-kinetic properties of fouling layers.

Streaming potential, which can be converted to zeta potential using the HelmholtzSmoluchowski (H-S) principle, ${ }^{16}$ can reflect the electrochemical properties of membrane. To 
date, streaming potential has been widely used to study electro-kinetic properties of (fouled) membrane due to its experimental simplicity. ${ }^{17}$ Soffer et al. ${ }^{18}$ applied an ex situ measurement to study the fouling of polysulfone UF membranes with different molecular weight cut off (MWCO). Streaming potential values were measured across the virgin and fouled membrane by varying the applied pressure $-\Delta P$ in the range of $0.1-0.5$ bar. They demonstrated that two fouling layers formed on both membranes had a different nature through coupling classical flux measurement with streaming potential analysis (results supported by microscopic pictures). They also concluded that a linear relationship existed between the change of zeta potential from clean membrane to fouled membrane and the degree of fouling.

Sung et $a l .{ }^{19}$ studied the evolution of the streaming potential coefficient during filtration of bovine serum albumin (BSA) suspension $\left(90 \mathrm{ppm}\right.$ in $1 \times 10^{-3} \mathrm{~mol} \mathrm{~L}^{-1} \mathrm{KCl}$ electrolyte, negatively charged at $\mathrm{pH}$ 6). They detected a decrease of the streaming potential coefficient from positive value to a negative one during filtration, which indicated that the electrostatic repulsion allowed the BSA particles existing mainly in the concentration polarization layer instead of being deposited onto the outer surface of the hollow fiber. Furthermore, the variations of the streaming potential were closely related to flux decline. The same phenomenon was also been reported by Benoit et al. ${ }^{20}$

In order to obtain electro-kinetic properties of fouling layer during its formation, Benoit et al. ${ }^{20}$ applied electrical potential measurements to monitor membrane fouling at constant pressure. Experimental results showed that the zeta potential values of clean and fouled membrane obtained by the new protocol were the same as the values obtained by classical streaming potential measurement. The results validated the new protocol that could be exploited during the growth of fouling layer on membrane surface.

Actually, existing method for monitoring fouled membrane by zeta potential, which was just applicable to the synthetic water (such as with single humic acid or BSA), ${ }^{21,22}$ was frequent in the literature. The study of zeta potential for monitor membrane fouling in surface water containing complex matters or the more complex effluent has not been mentioned before. Here, the apparent zeta potential of fouling layer are investigated for membrane fouling in-line coagulation/flocculation ultrafiltration (C/F-UF). The focus of this research was to exploring the relationship between apparent zeta potential of membrane and TMP during the membrane fouling as well as establishing functional relation between membrane fouling and apparent zeta potential. 


\section{Theoretical background}

The streaming potential is one of the most convenient techniques for assessing the charge properties of the pore surfaces of porous media. ${ }^{23-26}$ When a pressure gradient is applied through a charged porous medium, the charges in the mobile part of the electrical double layer are carried toward the low pressure side resulting in an electrical current in the direction of the flow, called the streaming current. The accumulation of charge at one end sets up an electric field which causes a conduction current in the opposite direction of the streaming current. When the conduction current equals the streaming current, a steady state is achieved. The resulting electrical potential difference that can be measured between the pore ends is the streaming potential $(\Delta \varphi)$. As shown in Fig. 1, the arrows perpendicular to the membrane surface is the direction of raw water, and the other arrow is the direction of permeate. The permeate was drawn out form membrane by using a peristaltic pump, and the colloidal particles was rejected by membrane pores. Hence, the fouling sludge was formed on its outer side, and the streaming potential is formed between cake layer and permeate. However, the ratio $(\Delta \varphi / \Delta P)_{I=0}$, called the streaming potential coefficient and it is a more informative quantity than the single streaming potential value, which depends on the pressure gradient applied through the porous medium. The streaming potential coefficient gives direct information about the electrostatic charge at the electrical double layer shear plane.

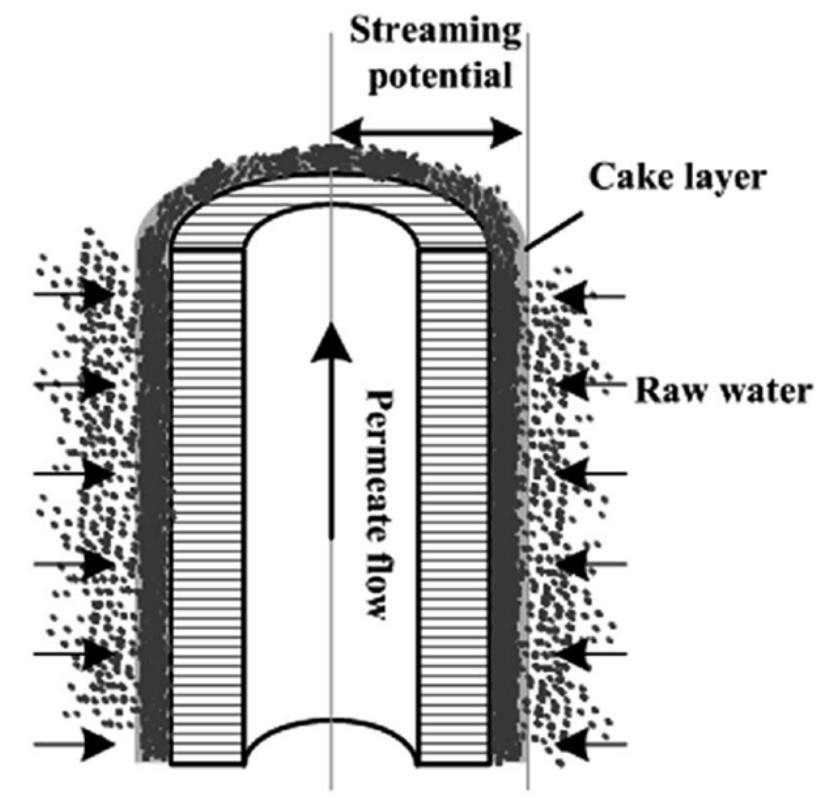

Fig. 1 The schematic diagram of streaming potential. 
Using streaming potential coefficient, apparent zeta potential can be calculated by the Helmholtz-Smoluchowski equation:

$$
\frac{\Delta \varphi}{\Delta P}=\frac{\varepsilon_{0} \varepsilon_{\mathrm{r}}}{\mu K} \xi
$$

where $\xi$ is the zeta potential, $\mathrm{mV} ; \varepsilon_{0} \varepsilon_{\mathrm{r}}$ is the permittivity of the solution in the pore, $\mathrm{C}$ $\mathrm{V}^{-1} \mathrm{~m}^{-1} ; \mu$ is the viscosity, $\mathrm{Pa} \mathrm{s}$; and $K$ is the conductivity of the electrolyte solution, $\mathrm{S} \mathrm{m}^{-1}$. Moreover, eqn (1) is restricted to the limit of $r_{\mathrm{p}} / \mathrm{k}^{-1}>10\left(r_{\mathrm{p}}\right.$ is pore radius, $\left.\mathrm{m}\right)$;

$$
\kappa=\sqrt{\varepsilon R T /\left(2 F^{2} c\right)}
$$

is Debye length, $\mathrm{m} ; F$ is Faraday constant, $\mathrm{C} \mathrm{mol}^{-1} ; c$ is the concentration in solution, mol $\mathrm{m}^{-3}, R$ is the molar gas constant, $\mathrm{J} \mathrm{mol}^{-1} \mathrm{~K}^{-1} ; T$ is the absolute temperature, $\mathrm{K}$ ).

In cases of membrane filtration in which the $\mathrm{H}-\mathrm{S}$ equation can be used, the Darcy law (just $R_{\mathrm{m}}$ and $R_{\mathrm{c}}$ were considered) (eqn (2)) may be combined with eqn (1), and the apparent zeta potential measured across the membrane can be written as a function of the permeate flow (eqn (3)):

$$
J=\frac{\Delta P}{\mu\left(R_{\mathrm{m}}+R_{\mathrm{c}}\right)}
$$

where $J$ is the permeate flux, $\mathrm{L} \mathrm{m}^{-3} \mathrm{~h}^{-1} ; P$ is the pressure across the membrane, $\mathrm{Pa} ; R_{\mathrm{m}}$ is the hydraulic resistance of the virgin membrane, $\mathrm{m}^{-1} ; R_{\mathrm{c}}$ is the hydraulic resistance of fouling layer, $\mathrm{m}^{-1}$.

$$
\xi_{\mathrm{a}}=\frac{\Delta \varphi K}{\varepsilon_{0} \varepsilon_{\mathrm{r}}\left(R_{\mathrm{m}}+R_{\mathrm{c}}\right) J}
$$

where $\xi_{\mathrm{a}}$ is the apparent zeta potential of the fouled membrane (membrane plus fouling layer) $(\mathrm{mV})$. When the flux permeate of membrane is kept as a constant, the instantaneous $\xi_{\mathrm{a}}$ can be calculated directly by measuring the streaming potential during the filtration.

\section{Materials and methods}

\subsection{Membrane}


All filtration tests were performed using polyvinylidene fluoride (PVDF) hollow fiber membrane (Tianjin MOTIMO Membrane Technology CO., LTD) with a mean pore size of about $0.1 \mu \mathrm{m}$. The membrane permeability, measured with deionized water (TOC $<0.1 \mathrm{mg}$ $\mathrm{L}^{-1}, \mathrm{pH} 6.5$ and conductivity of around $1 \mu \mathrm{S} \mathrm{cm}^{-1}$ ), was $20 \mathrm{~L} \mathrm{~m}^{-2} \mathrm{~h}^{-1}$ at the pressure of 20 $\mathrm{kPa}$. The filtration surface area was $0.01 \mathrm{~m}^{2}$. A new membrane was used for each filtration test.

\subsection{Raw water}

The surface water of Luanhe river was chosen as raw water in this study. As shown in Table 1, the raw water contained suspended colloid matters with negative charge, has a zeta potential of about $-21.5 \pm 0.8 \mathrm{mV}$ (measured at $\mathrm{pH} 6.5$ ). The hydrodynamic diameter of colloidal particles in raw water was measured at $20 \pm 1{ }^{\circ} \mathrm{C}$ by dynamic light scattering using a Nanosizer 1000 (Malvern, UK). Those colloidal particles exhibited a volume median diameter equal to $225 \pm 40 \mathrm{~nm}$ (Fig. 2). Based on the membrane pore size $(0.1 \mu \mathrm{m})$ and volume median diameter, total rejection of the colloidal particles was expected during filtration.

Table 1 Characteristics of natural surface water

\begin{tabular}{lll}
\hline Parameter & Unit & Value \\
\hline $\mathbf{p H}$ & - & $6.5 \pm 0.53$ \\
DOC & $\mathrm{mg} \mathrm{L}$ & $6.65 \pm 0.38$ \\
Turbidity & $\mathrm{NTU}$ & $3.64 \pm 0.44$ \\
Zeta potential & $\mathrm{mV}$ & $-21.5 \pm 0.82$ \\
Conductivity & $\mu \mathrm{S} \mathrm{cm}$ & $56.5 \pm 1.2$ \\
$\mathbf{C l}^{-}$ & $\mathrm{mg} \mathrm{L}^{-1}$ & $150.36 \pm 0.16$ \\
$\mathbf{N O}_{3}^{-}$ & $\mathrm{mg} \mathrm{L}^{-1}$ & $22.25 \pm 0.14$ \\
$\mathbf{S O}_{3}{ }^{--}$ & $\mathrm{mg} \mathrm{L}^{-1}$ & $9.68 \pm 0.08$ \\
$\mathbf{I}^{-}$ & $\mathrm{mg} \mathrm{L}^{-1}$ & $1.08 \pm 0.01$ \\
\hline
\end{tabular}




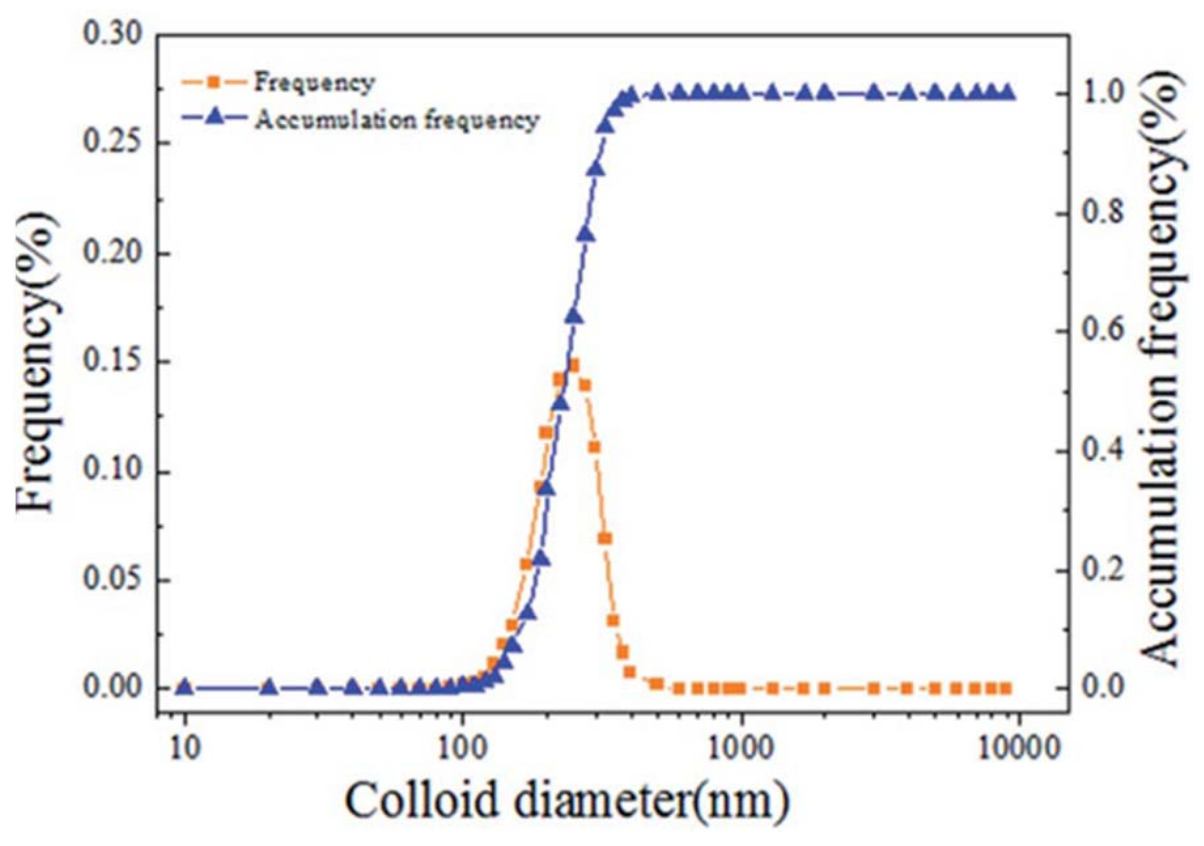

Fig. 2 The distribution of colloidal particle in raw water.

\subsection{Coagulant}

Although colloidal particles in raw water with negative surface charges can stay stable, they can be destabilized by adding coagulant. In this study, $\mathrm{FeCl}_{3}$ was chosen as the coagulant due to its common use and ability to remove suspended matters in micro-polluted water treatment.

\subsection{Filtration setup}

Compared with UF alone, coagulation as a pre-treatment process for UF could significantly enhance the flux recovery for surface water treatment. However, since most studies on membrane fouling control were conducted in a constant pressure mode and all full-scale membrane filtration plants are operated in constant-flux mode with regular backwashing, the results from these studies are not always representative for full-scale operations. Therefore, the membrane filtration setup in this study was designed at constant flux $20 \mathrm{~L} \mathrm{~m}^{-2} \mathrm{~h}^{-1}$ with constant backwashing flow by using peristaltic pumps (see in Fig. 3). C-UF experiments were carried out in a dead-end mode. The operating temperature was $20^{\circ} \mathrm{C}$ and the backwashing flux was two times higher than the permeate flux. 


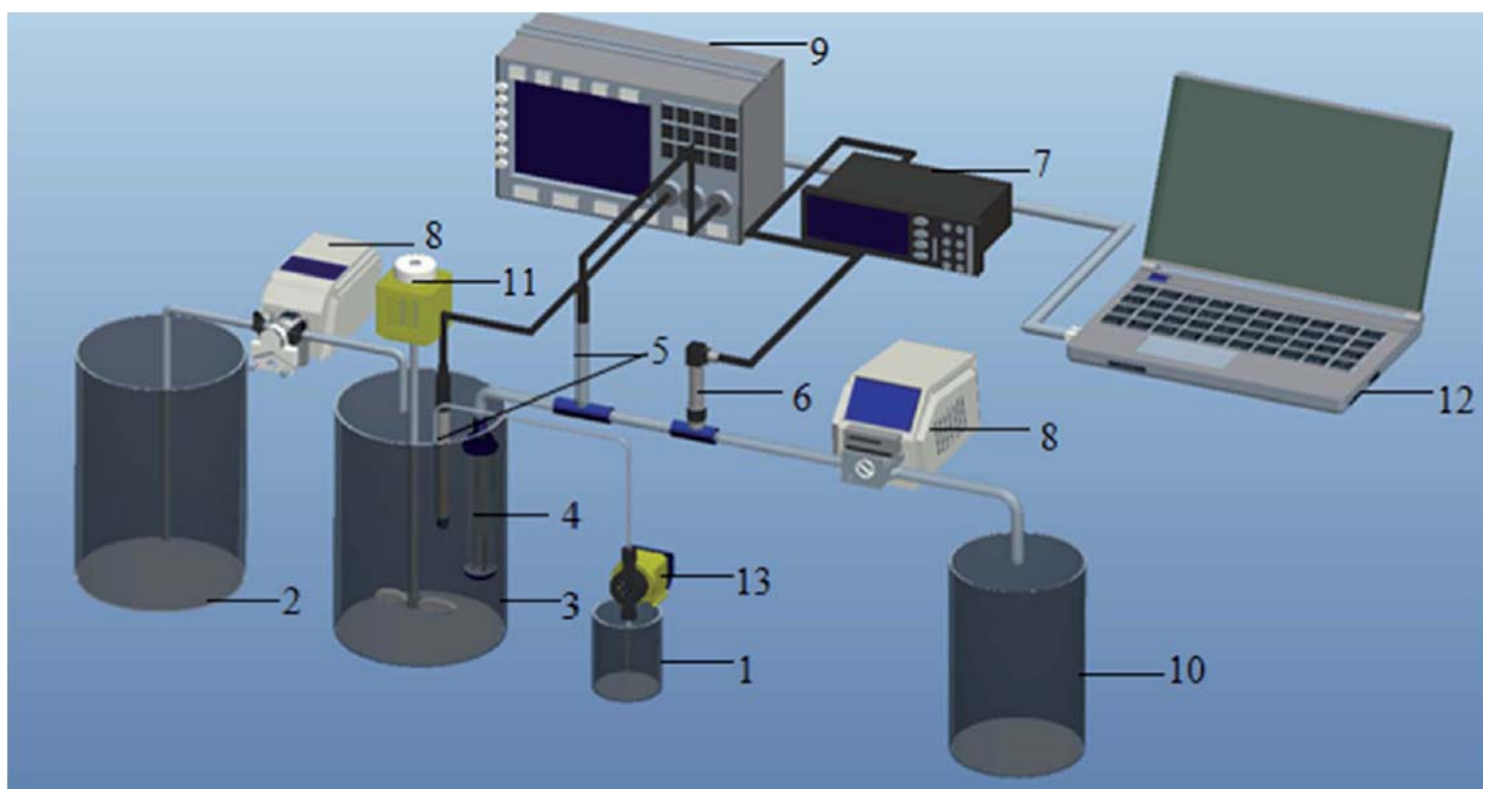

Fig. 3 Schematic diagram of the experiment setup for the streaming potential measurements of hollow fibers membrane model. ((1) - coagulant solution; (2) - raw water; (3) coagulation tank; (4) - membrane model; (5) -electrodes; (6) - pressure transmitter; (7) paperless recording;(8) - peristaltic pump; (9) - oscilloscope; (10) - permeate tank;(11) agitator paddle; (12) - computer; (13) - dosing pump)

\subsection{Measurement method of streaming potential}

Measurements of streaming potential and TMP across the membrane pores were performed, as shown in Fig. 3. The liquid was imposed through the membrane pore by a peristaltic pump. The streaming potential across the membranes were measured with a pair of commercial $\mathrm{Ag} / \mathrm{AgCl}$ reference electrodes placed on both permeate and retentate sides of the membrane module. Due to the raw water and the coagulant were added to coagulation tank at the same time with a certain ratio, the $\mathrm{FeCl}_{3}$ concentration in coagulation tank was constant value. So, without thinking of the effect of $\mathrm{FeCl}_{3}$ addition to streaming potential change, the potential difference between the electrodes was measured and displayed on an oscilloscope (Agilent, 54641A, USA).

\subsection{Analytical instruments}

The pressure difference of the membrane was measured using a pressure transmitter (Danfoss, MBS 3000, Denmark) which was connected to a paperless recording instrument (LCKLY, XSW10R, China). The zeta potential and diameter of colloid particles in raw water were determined by a Master Nanosizer 1000 (Malvern Instruments, UK). Dissolved organic 
carbon of water samples, turbidity, conductivities, $\mathrm{pH}$ were measured using a combustiontype organic carbon analyzer (TOC- $\mathrm{V}_{\mathrm{cph}}$ analyzer Shimadzu, Japan), an ultraviolet spectrophotometer (T6PERSEE, China), turbidimeter (HACH 2100N, USA), a conductivity meter (DDSJ-308A, China), a pH meter (PHS-3C, China), respectively.

\section{Results and discussion}

\subsection{Effect of $\mathbf{p H}$ on zeta potential of raw water and virgin membrane}

In order to understand and find out the influence of $\mathrm{pH}$ on zeta potential, measurements were made in the $\mathrm{pH}$ range of 1.5-9.5. Fig. 4 shows the variation of zeta potential of the raw water and the clean membrane as a function of $\mathrm{pH}$. The zeta potential of membrane was acquired in $0.001 \mathrm{M} \mathrm{KCl}$ solution by measuring streaming potential, whose applied pressure $-\Delta P$ in the range of $10-50 \mathrm{kPa}$ for at least 4 values. Each measurement was repeated at least five times on all samples.

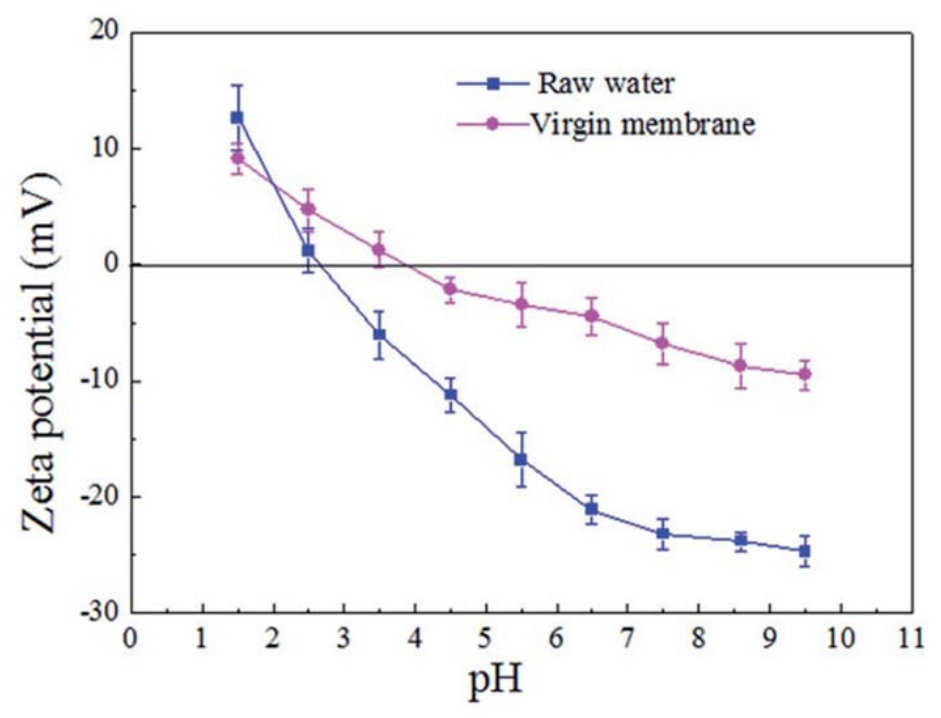

Fig. 4 The zeta potential variations of raw water and virgin membrane versus to $\mathrm{pH}$.

As shown in Table 1, the zeta potential of the raw water was nearly $-21.5 \mathrm{mV}$ at $\mathrm{pH}$ of approximately 6.5 (Fig. 4). As for the virgin membrane, the zeta potential was $-4.5 \mathrm{mV}$ at $\mathrm{pH}$ of 6.5 and was less negative compared to the raw water. The $\mathrm{pH}$ of iso-electric point for raw water was 2.9 , while the virgin membrane was 4.2 .

It was clear that the zeta potentials of the raw water and the virgin membrane were significantly influenced by the change of $\mathrm{pH}$. As the $\mathrm{pH}$ increased from 1.5 to 9.5 , the zeta 
potential of the raw water decreased from 12.6 to $-26.7 \mathrm{mV}$, while that of the virgin membrane decreased from 9.1 to $-9.5 \mathrm{mV}$, respectively. Thus, the $\mathrm{pH}$ of the feed water for membrane filtration was kept stable at 6.5 to avoid any effect on the measurement of zeta potential.

\subsection{Effect of coagulant dosage on zeta potential}

In this study, zeta potential was measured to determine the optimum coagulant dosage at $\mathrm{pH}$ 6.5 and the results are shown in Fig. 5. Dosages of coagulant $\left(\mathrm{FeCl}_{3}\right)$ ranged from $0 \mathrm{mg} \mathrm{L}^{-1}$ to $30 \mathrm{mg} \mathrm{L}^{-1}$.

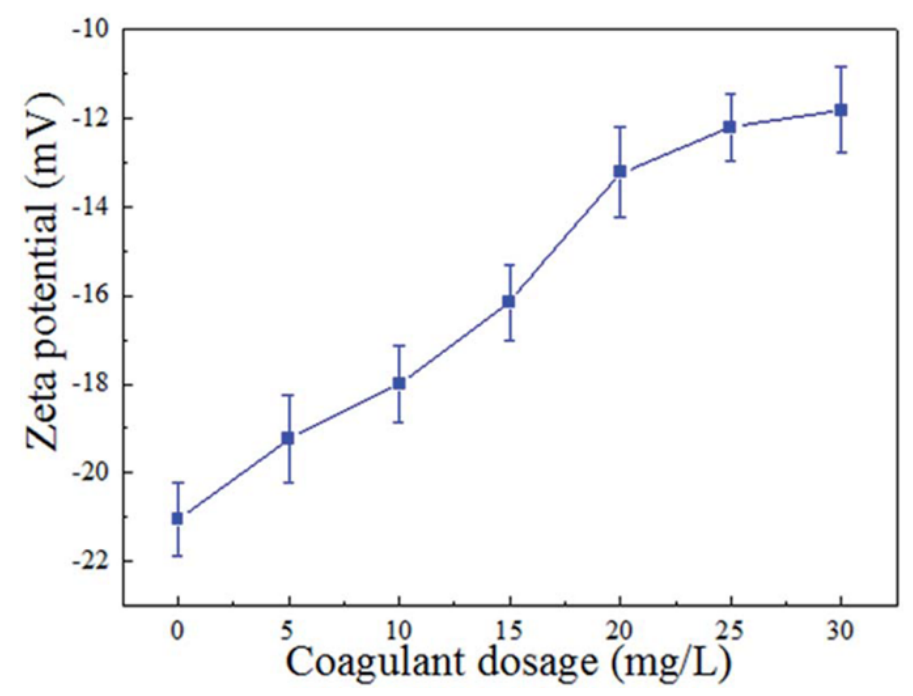

Fig. 5 Influence of coagulant dosage on the zeta potentials of colloidal particle. The $\mathrm{pH}$ of the flocculated water was kept stabilized around 6.5 by $\mathrm{NaH}_{2} \mathrm{PO}_{4} / \mathrm{Na}_{2} \mathrm{HPO}_{4}$ buffer solution.

Overall, the zeta potential of particles increased with the increase of coagulant dosage. It increased rapidly (from $-21.5 \mathrm{mV}$ to $-13.2 \mathrm{mV}$ ) when coagulant dosage increased from 0 to $20 \mathrm{mg} \mathrm{L}^{-1}$, and then approached a plateau at higher dosages. Hence, $20 \mathrm{mg} \mathrm{L}^{-1}$ was the optimum dose in terms of charge neutralization.

\subsection{Apparent zeta potential and TMP variation during in-line filtration at constant flux}

In this study, in-line coagulation without settling, as a pre-treatment to ultrafiltration processes, was carried out for micro-polluted water treatment. As the permeate flux of membrane was kept as a constant during the filtration, an increase in TMP (because of membrane fouling) was observed. 
In order to account for the behavior of fouling layer of membrane in C-UF, the streaming potential across the membrane was measured in-line during the filtration process. The direct filtration of raw water was also carried out for comparison.

As can be seen in Fig. 6a and b, the filtration of two suspensions (the raw water and the coagulation mixture) led to a gradual increase of the TMP. The TMP rise was about 55 $\mathrm{kPa}$ with colloidal particles deposited on membrane surface during the filtration of raw water, while there was only $40 \mathrm{kPa}$ for C-UF process. With regard to the apparent zeta potential, an obvious difference was observed between the two membranes.

As already given in Fig. 4, the hollow fiber membrane was negatively charged at $\mathrm{pH}$ 6.5, while the colloidal matters were more negatively charged. Fig. 6 shows the absolute values of the apparent zeta potential of membrane in the process of direct filtration was higher than those of the membrane in C-UF, which were consistent with TMP in both processes.

In C-UF process, the apparent zeta potential changed a little within the first $50 \mathrm{~min}$ filtration, and it indicated that the properties of the charged membrane didn't changed during the filtration process possibly due to the deposition of electrically neutral flocs onto the membrane surface. However, the absolute value of apparent zeta potential increased as the filtration proceeded and even a faster increase rate appeared during 200-600 min. This change was possibly due to the deposition of colloidal particles onto the outer surface of the hollow fiber.

The negatively charged virgin membrane $\left(\xi_{\mathrm{m}}=-4.5 \mathrm{mV}\right)$ was ultimately overwhelmed by the more negatively charged colloidal matters resulting from the flocculated water. The membrane being negatively charged after fouled in coagulation mixture which $\xi_{\mathrm{m}}$ was kept at $-10.5 \mathrm{mV}$. It was because the repulsive electrostatic interaction makes the colloidal particles stay in a suspended state above the outer surface of hollow fibers instead of being deposited in fouling layer. However, the apparent zeta potential of fouled membrane in direct UF was $-15.6 \mathrm{mV}$. This difference meant that the apparent zeta potential of the fouling membrane in C-UF with colloidal particles deposition was lower (in absolute value) than that of the membrane in direct filtration of raw water. 

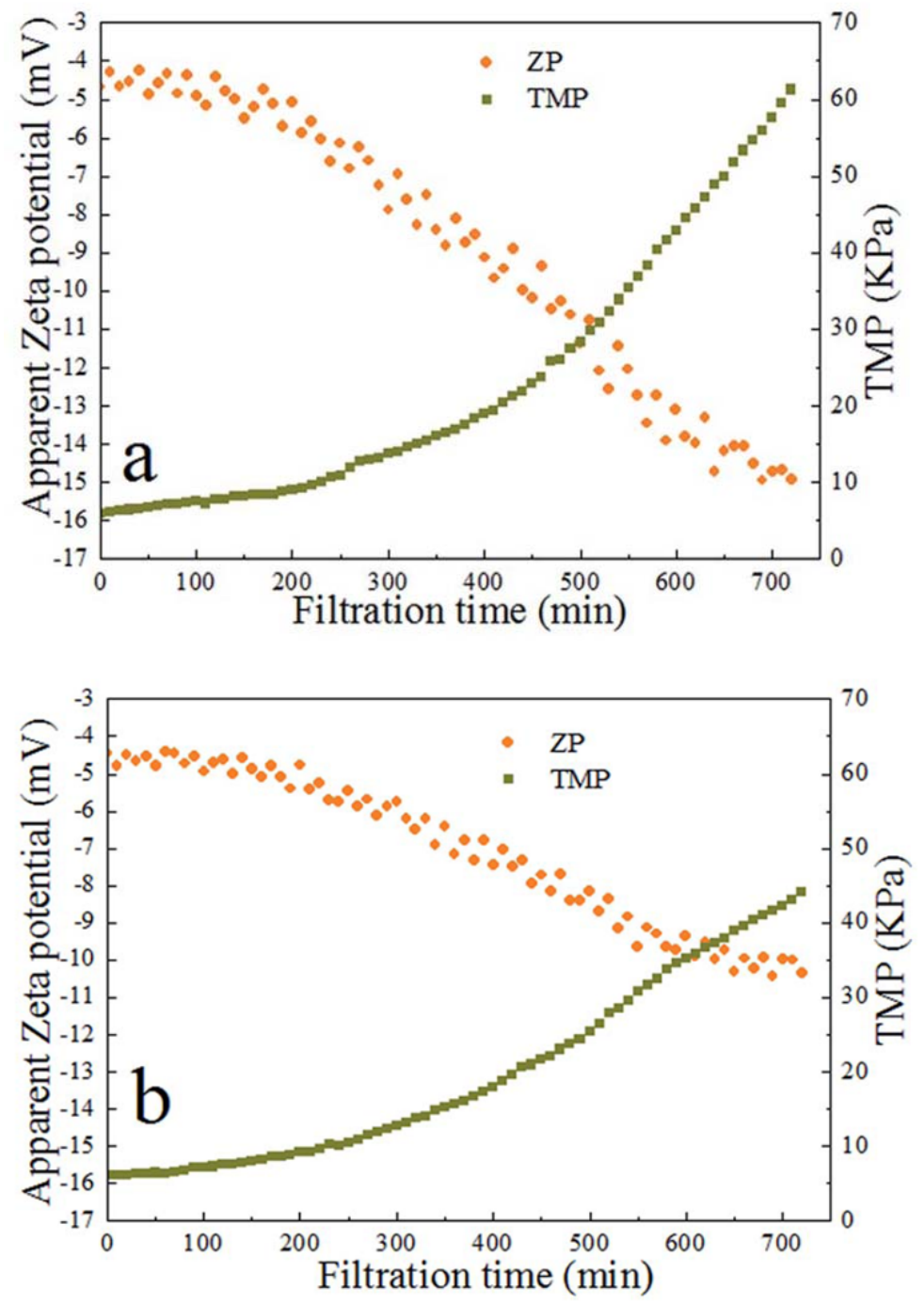

Fig. 6 Apparent zeta potential and TMP versus filtration time during filtration of (a) the raw water directly without any pretreatment and (b) coagulation suspension of the raw water coagulated with $\mathrm{FeCl}_{3}$ at the dosage of $20 \mathrm{mg} \mathrm{L}^{-1}$. Both of the filtration were operated at a constant flux of $20 \mathrm{~L} \mathrm{~m}^{-2} \mathrm{~h}^{-1}$. The buffer solution $\left(\mathrm{Na}_{2} \mathrm{HPO}_{4} / \mathrm{KH}_{2} \mathrm{PO}_{4}\right)$ was applied to stabilize $\mathrm{pH}$ at 6.5 .

\subsection{Apparent zeta potential with the variation of resistance}

The relationship between the apparent zeta potential of fouled membrane and the variation of filtration resistance was considered. The resistance was calculated on the basis of the Darcy's law using TMP and membrane flux. According to the date got from Fig. 6, the function relationship of apparent zeta potential and filtration resistance was established by curve fitting. The non-linear equations were conformed to the form of Michaelis-Menten equation. The correlation coefficients of the curve in raw water direct membrane filtration process and 
C-UF process were 0.947 and 0.934 , respectively (see in Fig. 7). It meant that the membrane fouling could be monitored by observing the apparent zeta potential intuitively.

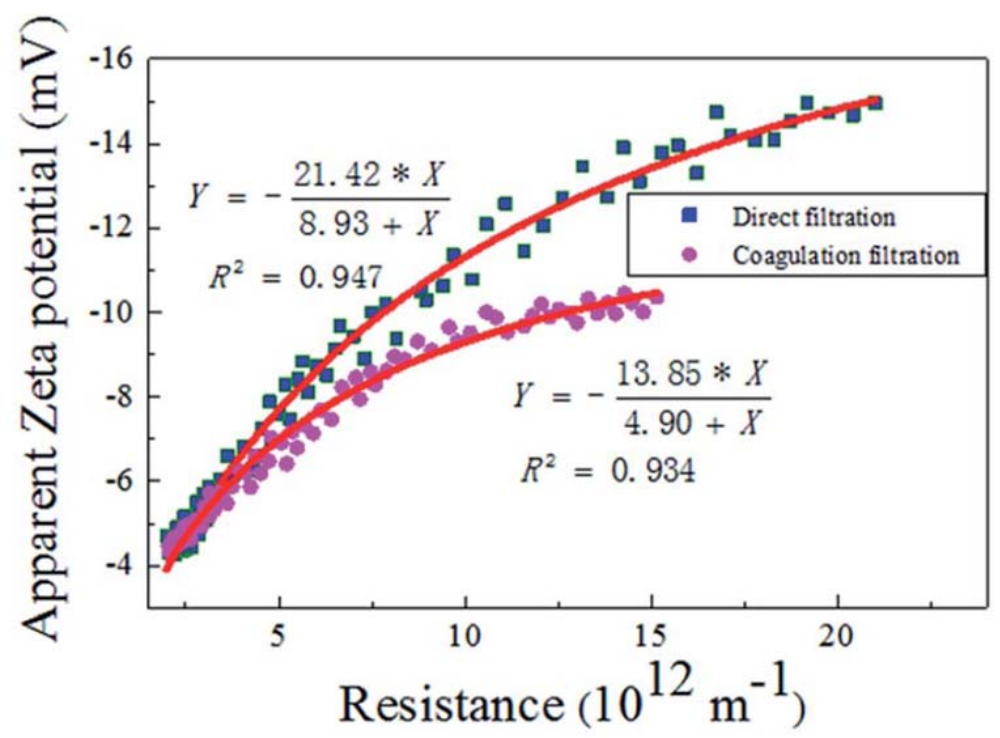

Fig. 7 The fitted curve between apparent zeta potential and membrane filtration resistance.

The apparent zeta potential was positive correlated with the filtration resistance at the beginning of filtration. This meant the disposition of colloidal particles is the major reason for membrane fouling. It was also clear that the apparent zeta potential of membrane in direct filtration process after long-running could be up to $-21.42 \mathrm{mV}$ which was close to zeta potential of raw water $(\xi=-21.5 \mathrm{mV})$, this phenomenon was probably due to the apparent zeta potential of fouled membrane is exactly the value of feed water when the membrane fouling is serious enough. The identical conclusion was also confirmed in the process of CUF. The apparent zeta potential of membrane in C-UF could be up to $-13.85 \mathrm{mV}$ which was close to zeta potential of coagulation mixture $(\xi=-13.2 \mathrm{mV})$. This is because the quality of raw water can influence the apparent zeta potential of fouled membrane in filtration process. Therefore, the measurement method, experimental results and the fitting function could provide references for the membrane fouling research.

\subsection{The effect of backwashing on the change of apparent zeta potential}

The changes of apparent zeta potential along with the periodical backwashing are shown in Fig. 8. When the TMP increased to $44.5 \mathrm{kPa}$ (the apparent zeta potential of fouled membrane reached a plateau at the same time), membrane backwashing was conducted with 
ultrapure water for $1 \mathrm{~min}$ under the pressure of $0.04 \mathrm{MPa}$ and aeration intensity of $500 \mathrm{~L}$ $\mathrm{m}^{-2} \min ^{-1}$. After two backwashing, the TMP of the fouled membrane model decreased to 8.7 and $12.6 \mathrm{kPa}$, respectively, indicating that a fraction of colloidal particles still remained on the membrane surface. The apparent zeta potential increased from -10.4 to $-5.7 \mathrm{mV}$, but was lower than the value of the virgin membrane $(\xi=-4.5 \mathrm{mV})$, suggesting that the apparent zeta potential was affected by the residual colloidal particles. After the second backwashing, the apparent zeta potential of fouled membrane was $-7.3 \mathrm{mV}$. The descending rate of the apparent zeta potential was getting larger, which was in accordance with the trend of TMP change.

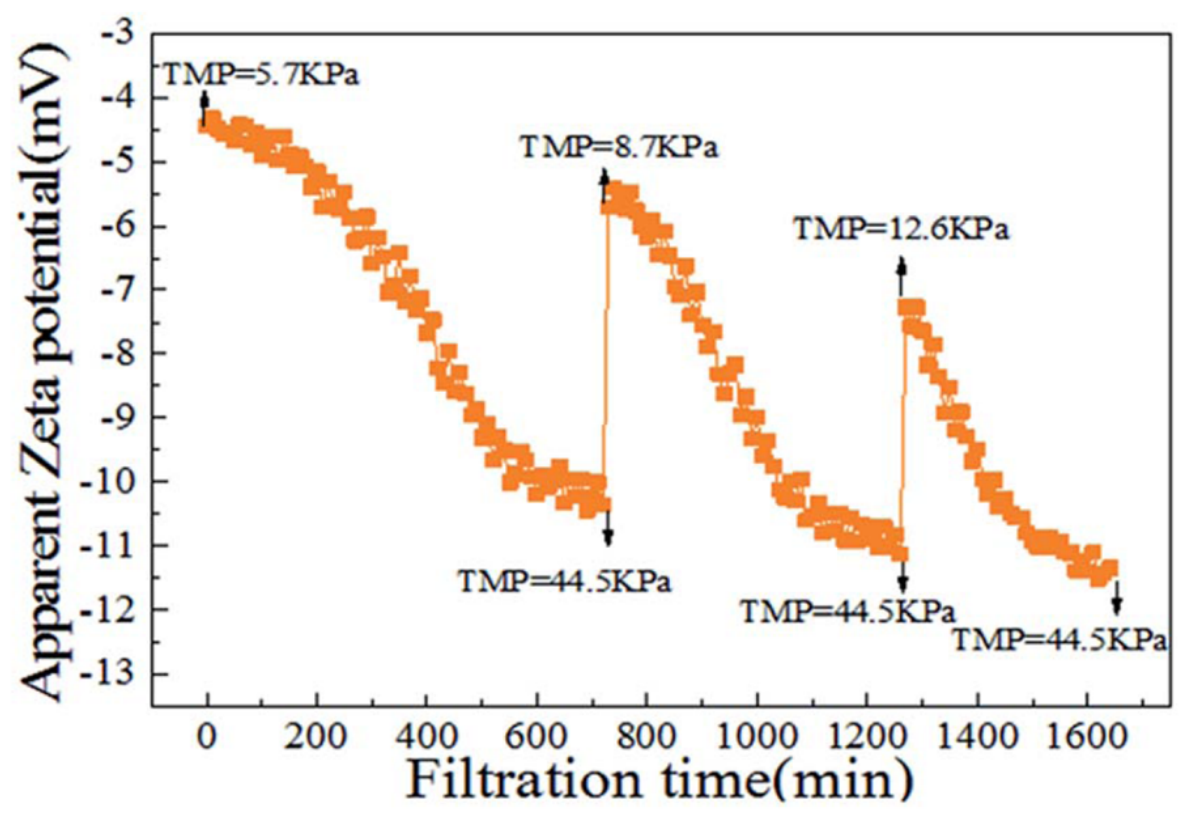

Fig. 8 The effect of backwashing on the apparent zeta potential of membrane. The backwashing was operated and last for $60 \mathrm{~s}$ when the TMP increased to $44.5 \mathrm{kPa}$.

After backwashing using demineralized water, the concentration of negatively charged colloidal particles around the membrane was reduced. However, the surface of membrane still retained certain negative charged colloidal particles from the feed water. Therefore, the negatively charged colloidal particles on the membrane remained and increased during the next filtration stage under the force of filtration and concentration difference. In the later stage of filtration the repulsive force between the negatively charged colloidal particles on membrane surface and colloidal particles in the feed water was completely restored. As a result, the apparent zeta potential reached a new level. Overall, apparent zeta potential could 
be a useful indicator for monitoring membrane fouling during micro-polluted water treatment (including filtration and backwashing).

\section{Conclusions}

This study aims to find a useful and simple method to monitor membrane fouling in micropolluted water treatment. The apparent zeta potential analysis, which presented the importance information on membrane surface charge, could be applied for membrane fouling monitoring.

In order to validate this method, the surface water of Luanhe river and PVDF hollow fiber ultrafiltration membranes have been used as the case study. Batch experiments indicated that both $\mathrm{pH}$ and coagulant dosage have great influence on zeta potential. Results of in-line coagulation show that the absolute values of apparent zeta potential were smaller than those of membrane in directed filtration process, which were consistent with TMP in both processes. The correlation coefficient between apparent zeta potential and TMP was 0.934 during C-UF process, the apparent zeta potential of membrane could close to the zeta potential of coagulation mixture after long-running. The information of both membrane filtration resistance and apparent zeta potential assists the further understanding of the membrane, its interactions with foulants, as well as fouling characterization. By observing the change of apparent zeta potential during membrane backwashing, the effect of backwashing could be reflected visually. In conclusion, the variations of apparent zeta potential were dependent on the fouling layer properties. Thus, the calculations of apparent zeta potential could be used to understand membrane as a fouling indicator.

\section{Acknowledgements}

This study was financially supported by the National Natural Science Foundation of China (No. 51378349), China Postdoctoral Science Foundation (2013M541184), and Program for Changjiang Scholars and Innovative Research Team in University of Ministry of Education of China (Grand No. IRT13084).

\section{References}

1. T. D. Waite, A. I. Schafer, A. G. Fane and A. Heuer, J. Colloid Interface Sci., 1999, 212, 264-274. 
2. S. H. Maruf, L. Wang, A. R. Greenberg, J. Pellegrino and Y. Ding, J. Membr. Sci., 2013, $428,598-607$.

3. H. Guo and J. Hu, Desalination, 2012, 290, 115-124.

4. X. Shi, R. Field and N. Hankins, Desalin. Water Treat., 2011, 35, 68-81.

5. S. Peldszus, C. Halle, R. H. Peiris, M. Hamouda, X. Jin, R. L. Legge, H. Budman, C. Moresoli and P. M. Huck, Water Res., 2011, 45, 5161-5170.

6. J. Mendret, C. Guigui, R. Schmitz and C. Cabassud, J. Membr. Sci., 2009, 333, 20-29.

7. X. Li, J. Li, J. Wang, H. Wang, B. He and H. Zhang, J. Membr. Sci., 2013, 444, 394-401.

8. X. Li, J. Li, J. Wang, H. Zhang and Y. Pan, J. Membr. Sci., 2012, 411, 137-145.

9. P. R. B'erub'e, G. Afonso, F. Taghipour and C. C. V. Chan, J. Membr. Sci., 2006, 279, 495-505.

10. J. Mendret, C. Guigui, P. Schmitz, C. Cabassud and P. Duru, AIChE J., 2007, 53, 22652274.

11. D. Hughes, U. K. Tirlapur, R. Field and Z. Cui, J. Membr. Sci., 2006, 280, 124-133.

12. A. Szymczyk, Y. I. Dirir, M. Picot, I. Nicolas and F. Barriere, J. Membr. Sci., 2013, 429, $44-51$.

13. G. Hurwitz, G. R. Guillen and E. M. V. Hoek, J. Membr. Sci., 2010, 349, 349-357.

14. M. Ernst, A. Bismarck, J. Springer and M. Jekel, J. Membr. Sci., 2000, 165, 251-259.

15. A. S. Al-Amoudi, Desalination, 2010, 259, 1-10.

16. H. Zeng, H. Ye, H. Liu and H. Xie, Desalination, 2006, 195, 226-234.

17. M. Wang, Q. An, L. Wu, J. Mo and C. Gao, Chin. J. Anal. Chem., 2007, 35, 605-610.

18. Y. Soffer, J. Gilron and A. Adin, Desalination, 2002, 146, 115-121.

19. J. H. Sung, M. S. Chun and H. J. Choi, J. Colloid Interface Sci., 2003, 264, 195-202.

20. B. Teychene, P. Loulergue, C. Guigui and C. Cabassud, J. Membr. Sci., 2011, 370, 45-57.

21. H. Dong, B. Gao, Q. Yue, S. Sun, Y. Wang and Q. Li, Chem. Eng. J., 2014, 258, 442-449.

22. L. Feng, W. Wang, R. Feng, S. Zhao, H. Dong, S. Sun and B. Gao, Chem. Eng. J., 2015, 262, 1161-1167.

23. Q. Yun-Ren and Q. Jing, J. Membr. Sci., 2013, 425-426, 71-76.

24. Y. Lanteri, P. Fievet, S. Deon, P. Sauvade, W. Ballout and A. Szymczyk, J. Membr. Sci., 2012, 411, 193-200.

25. C. Werner, H. J. Jacobasch and G. Reichelt, J. Biomater. Sci., Polym. Ed., 1995, 7, 61-76.

26. P. Fievet, M. Sbai, A. Szymczyk and A. Vidonne, J. Membr. Sci., 2003, 226, 227-236. 